\title{
Existence and Uniqueness of Weak Solutions for Novel Anisotropic Nonlinear Diffusion Equations Related to Image Analysis
}

\author{
Anas Tiarimti Alaoui $(i)$ and Mostafa Jourhmane \\ TIAD Laboratory, Department of Mathematics, Faculty of Sciences and Technology, Sultan Moulay Slimane University, \\ Beni Mellal 23000, Morocco
}

Correspondence should be addressed to Anas Tiarimti Alaoui; a.tiarimti@usms.ma

Received 16 January 2021; Revised 16 February 2021; Accepted 17 February 2021; Published 8 March 2021

Academic Editor: Sun Young Cho

Copyright ( $) 2021$ Anas Tiarimti Alaoui and Mostafa Jourhmane. This is an open access article distributed under the Creative Commons Attribution License, which permits unrestricted use, distribution, and reproduction in any medium, provided the original work is properly cited.

This paper establishes the existence and uniqueness of weak solutions for the initial-boundary value problem of anisotropic nonlinear diffusion partial differential equations related to image processing and analysis. An implicit iterative method combined with a variational approach has been applied to construct approximate solutions for this problem. Then, under some a priori estimates and a monotonicity condition, the existence of unique weak solutions for this problem has been proven. This work has been complemented by a consistent and stable approximation scheme showing its great significance as an image restoration technique.

\section{Introduction}

In the last three decades, nonlinear diffusion equations have inspired numerous research studies in various application ranges. Perona and Malik [1] were the first to introduce such equation in image processing and analysis in the following manner:

$$
\begin{cases}\frac{\partial u}{\partial t}-\nabla \cdot[c(|\nabla u|) \nabla u]=0, & \text { in } \Omega \times(0, T], \\ \langle c(|\nabla u|) \nabla u, \mathbf{n}\rangle=0, & \text { on } \partial \Omega \times(0, T], \\ u(x ; 0)=u_{0}(x), & \text { in } \Omega,\end{cases}
$$

where $\Omega$ is an image domain in $\mathbb{R}^{2}$ and $c$ is a positive decreasing function defined on $\mathbb{R}_{+}$.

When it comes to processing a digital image, Perona and Malik chose the above model to preserve meaningful features such as edges while reducing irrelevant information such as noise in the homogeneous area. Nevertheless, this model, known as an isotropic nonlinear diffusion equation, handles an image feature with the same amount of blurring in all its directions. For instance, this process cannot successfully eliminate noises at edges [2]. Accordingly, it might be wise to consider the orientation of essential features by using anisotropic diffusion. Weickert [2] introduced this property by defining an orientation descriptor using the structure tensor, which is convenient to identify features such as corners and T-junctions. Besides, digital images present some structural difficulties; that is, they are discrete in space and image intensity values. Accordingly, it would be of great interest to adapt the diffusion to digital images' structure by considering vertical, horizontal, and diagonal differential operators. Due to these reasons, we modeled and developed anisotropic nonlinear diffusion equations using a novel diffusion tensor.

Various tools can be used to examine the existence of solutions for nonlinear partial differential equations (PDEs), such as variational techniques, monotonicity method, fixedpoint theorems, iterative methods, and truncation techniques; for more detailed information, we refer to [3-7] and the references therein. These PDEs have been motivated by various applications such as image restoration and reconstruction (see, for example, [3, 4, 8-11]). Moreover, the 
image processing of the brain allows the localization of epileptogenic foci for the patient. A noninvasive method has been examined numerically as an inverse problem in [12].

Under some challenging conditions, the existence and uniqueness of weak solutions for the Perona and Malik model have been investigated in the bounded variation space $B V(\Omega)[3,13]$. In some other functional frameworks, Wang and Zhou have thoroughly studied in [4] and proved the existence and uniqueness of weak solutions in the Orlicz space $L \log L(\Omega)$ using a new diffusion function $c(s)=((s+$ $(s+1) \log (s+1)) /(s(s+1)))$ for all $s \geq 0$.

In this paper, we suppose that $\Omega$ is an open-bounded domain of $\mathbb{R}^{2}$ with Lipschitz boundary $\partial \Omega$, and $T$ is a positive number. We denote

$$
\left\{\begin{array}{l}
\partial_{x_{1}} u:=u_{x_{1}}:=\nabla u \cdot \mathbf{e}_{1}, \\
\partial_{x_{2}} u:=u_{x_{2}}:=\nabla u \cdot \mathbf{e}_{2}, \\
\partial_{x_{12}} u:=u_{x_{12}}:=\nabla u \cdot \frac{\mathbf{e}_{1}+\mathbf{e}_{2}}{\left|\mathbf{e}_{1}+\mathbf{e}_{2}\right|} \\
\partial_{x_{-12}} u:=u_{x_{-12}}:=\nabla u \cdot \frac{-\mathbf{e}_{1}+\mathbf{e}_{2}}{\left|-\mathbf{e}_{1}+\mathbf{e}_{2}\right|}
\end{array}\right.
$$

where $\left(e_{1}, e_{2}\right)$ is the canonical basis of $\mathbb{R}^{2}$. We consider the following anisotropic nonlinear parabolic initial-boundary value problem:

$$
\begin{cases}\frac{\partial u}{\partial t}-\nabla \cdot\left[\mathbf{D}_{\nabla u} \nabla u\right]=0, & \text { in } \Omega \times(0, T], \\ \left\langle\mathbf{D}_{\nabla u} \nabla u, \mathbf{n}\right\rangle=0, & \text { on } \partial \Omega \times(0, T], \\ u(x ; 0)=u_{0}(x), & \text { in } \Omega,\end{cases}
$$

where $\mathbf{D}_{\nabla u}$, the diffusion tensor, is a real symmetric positive definite matrix of $\mathbb{R}^{2 \times 2}$ defined as follows:

$$
\begin{aligned}
& \mathbf{D}_{\nabla u}=\left(\begin{array}{cc}
g\left(\left|u_{x_{1}}\right|\right)+\frac{g\left(\left|u_{x_{12}}\right|\right)+g\left(\left|u_{x_{-12}}\right|\right)}{2} & \frac{g\left(\left|u_{x_{12}}\right|\right)-g\left(\left|u_{x_{-12}}\right|\right)}{2} \\
\frac{g\left(\left|u_{x_{12}}\right|\right)-g\left(\left|u_{x_{-12}}\right|\right)}{2} & g\left(\left|u_{x_{2}}\right|\right)+\frac{g\left(\left|u_{x_{12}}\right|\right)+g\left(\left|u_{x_{-12}}\right|\right)}{2}
\end{array}\right), \\
& \phi(s)=\int_{0}^{s} r g(r) \mathrm{d} r, \quad s \geq 0,
\end{aligned}
$$

and $g: \mathbb{R}_{+} \longrightarrow \mathbb{R}$ is a $C^{1}$ positive decreasing function. Then, we can define $\phi: \mathbb{R}_{+} \longrightarrow \mathbb{R}$ as a $C^{2}$ function such that 


$$
\left\{\begin{array}{l}
\phi(0)=\phi^{\prime}(0)=0, \phi(s)>0, \phi^{\prime}(s)>0, \quad \text { for } s \in \mathbb{R}_{+}^{*}, \\
\phi^{\prime \prime}(s) \geq 0, s \phi^{\prime \prime}(s) \leq \phi^{\prime}(s), \quad \text { for } s \in \mathbb{R}_{+}, \\
0<\lim _{s \longrightarrow \infty} \frac{\phi(s)}{s \log (s)}<\infty, 0<\lim _{s \longrightarrow \infty} \frac{\phi^{\prime}(s)}{\log (s)}<\infty, \\
\lim _{s \longrightarrow 0^{+}} \frac{\phi^{\prime}(s)}{s}>0, \lim _{s \longrightarrow \infty} \frac{\phi^{\prime}(s)}{s}=0 .
\end{array}\right.
$$

To construct an adaptive diffusion tensor, the function $g$ is approximated numerically by a cubic Hermite spline [14] that interpolates numeric data specified at $0=k_{0}<k_{1}<\cdots<k_{m}$ with $m \in \mathbb{N}^{*}$ :

(6)

$$
g(s)= \begin{cases}p_{k_{i}} P_{1, k_{i} k_{i+1}}(s)+v_{k_{i}} P_{2, k_{i} k_{i+1}}(s)+p_{k_{i+1}} P_{1, k_{i+1} k_{i}}(s)+v_{k_{i+1}} P_{2, k_{i+1} k_{i}}(s), & s \in\left[k_{i}, k_{i+1}[\right. \\ p_{k_{m}} g_{k_{m}, 1}(s)+v_{k_{m}} g_{k_{m}, 2}(s), & i \in\{0,1, \ldots, m-1\}, \\ & s \in\left[k_{m}, \infty[\right.\end{cases}
$$

where $p$. and $v$. are the coefficients used to define the po-

And we may consider sition and the velocity vector at a specific point, $k_{i}$ are the threshold parameters, $\left\{P_{j, c d}\right\}$ is the family of the basis functions composed of polynomials of degree 3 used on the interval $[c, d[$ such that

$$
\left\{\begin{array}{l}
P_{1, c d}(s)=\frac{(s-d)^{2}(2 s+d-3 c)}{(d-c)^{3}} \\
P_{2, c d}(s)=\frac{(s-d)^{2}(s-c)}{(d-c)^{2}}
\end{array}\right.
$$

$$
\left\{\begin{array}{l}
g_{k_{m}, 1}(s)=\frac{k_{m}}{\log \left(k_{m}\right)+2} \frac{2 s(\log (s)+1)-k_{m} \log \left(k_{m}\right)}{s^{2}}, \\
g_{k_{m}, 2}(s)=\frac{k_{m}^{2}}{\log \left(k_{m}\right)+2} \frac{s(\log (s)+1)-k_{m}\left(\log \left(k_{m}\right)+1\right)}{s^{2}} .
\end{array}\right.
$$


From the definition of $\phi$, we can deduce

$$
\phi(s)= \begin{cases}C_{i}+\sum_{j=0}^{3} \frac{A_{k_{i} k_{i+1}, j} s^{j+2},}{j+2} & s \in\left[k_{i}, k_{i+1}[, i \in\{0,1, \ldots, m-1\},\right. \\ A_{k_{m}, 2} s \log (s)+A_{k_{m}, 1} \log (s)+C_{m}, & s \in\left[k_{m}, \infty[,\right.\end{cases}
$$

where $C_{i}$ and $C_{m}$ are constants determined by the continuity of $\phi$ at each $k_{i}$. In this case, the values of the coefficients $A_{k_{i} k_{i+1}, j}$ are determined experimentally provided that $\phi$ satisfying the above conditions on $\left[0, k_{m}\right.$ [. Besides, we may introduce some sufficient conditions on $k_{m}$ and $A_{k_{m} \text {. }}$ that guarantee the properties of $\phi$ on $\left[k_{m}, \infty[\right.$ :

$$
\left\{\begin{array}{l}
k_{m} \geq 1, \\
A_{k_{m}, 2}>0 \\
A_{k_{m}, 1}<k_{m} A_{k_{m}, 2} \\
A_{k_{m}, 1} \geq-\frac{k_{m} \log \left(k_{m}\right)}{2} A_{k_{m}, 2} .
\end{array}\right.
$$

Anisotropic diffusion model (3) allows strong directional smoothing within the areas where $\left|u_{x_{1}}\right|,\left|u_{x_{2}}\right|,\left|u_{x_{12}}\right|$, or $\left|u_{x_{-12}}\right|$ is small and prevents blurring boundaries, contours, or corners that separate neighboring areas, where one or a combination of these differential operators has significant value.

Moreover, the matrix $\mathbf{D}_{\nabla u}$ has two eigenvalues $\lambda_{+/-}$:

$$
\begin{aligned}
\lambda_{+/-}= & \frac{1}{2}\left(g\left(\left|u_{x_{1}}\right|\right)+g\left(\left|u_{x_{2}}\right|\right)+g\left(\left|u_{x_{12}}\right|\right)+g\left(\left|u_{x_{-12}}\right|\right)\right. \\
& \left. \pm \sqrt{\left(g\left(\left|u_{x_{1}}\right|\right)-g\left(\left|u_{x_{2}}\right|\right)\right)^{2}+\left(g\left(\left|u_{x_{12}}\right|\right)-g\left(\left|u_{x_{-12}}\right|\right)\right)^{2}}\right),
\end{aligned}
$$

with $\theta_{+/-}$are the corresponding eigenvectors. We can then expand the first equation of (3) into

$$
\frac{\partial u}{\partial t}=\nabla \cdot\left[\lambda_{+} \theta_{+} \theta_{+}^{T} \nabla u\right]+\nabla \cdot\left[\lambda_{-} \theta_{-} \theta_{-}^{T} \nabla u\right] .
$$

Accordingly, it is clear from the expression of $\lambda_{+/-}$that $\lambda_{+} \geq \lambda_{-}>0$, which means that the diffusion towards $\theta_{+}$is privileged over $\theta_{-}$. In fact, the difference $\left(\lambda_{+}-\lambda_{-}\right)^{2}=\left(g\left(\left|u_{x_{1}}\right|\right)-g\left(\left|u_{x_{2}}\right|\right)\right)^{2}+$

$\left(g\left(\left|u_{x_{12}}\right|\right)-g\left(\left|u_{x_{-12}}\right|\right)\right)^{2}$ indicates the isotropic diffusion for zero value and anisotropic diffusion for positive values.

Henceforth, we will assume that the initial value satisfies

$$
u_{0} \in L^{2}(\Omega)
$$

and we will introduce the following Orlicz space:

$$
L \log L^{k_{m}}(\Omega)=\left\{u: \Omega \longrightarrow \mathbb{R}\left|\int_{\Omega \cap\left\{|u| \geq k_{m}\right\}}\right| u \mid \log (|u|) \mathrm{d} x<\infty\right\}
$$

Next, we define weak solutions for problem (3) on $Q_{T}:=\Omega \times(0, T]$ with $T>0$ :

Definition 1. A function $u: \bar{Q}_{T} \longrightarrow \mathbb{R}$ is a weak solution for problem (3) if the following conditions are satisfied:

(i) $u \in C\left([0, T] ; L^{2}(\Omega)\right) \cap L^{1}\left(0, T ; W^{1,1}(\Omega)\right) \quad$ with $\partial_{x_{i}} u \in L \log L^{k_{m}}(\Omega)$ for $i=1,2$. (ii) For any $\varphi \in C^{1}\left(\bar{Q}_{T}\right)$ with $\varphi(., T)=0$, we have

$$
-\int_{\Omega} u_{0}(x) \varphi(x, 0) \mathrm{d} x+\int_{0}^{T} \int_{\Omega}\left[-u \varphi_{t}+\mathbf{D}_{\nabla u} \nabla u \cdot \nabla \varphi\right] \mathrm{d} x \mathrm{~d} t=0 .
$$

Now, we state our main theorem.

Theorem 1. Under assumption (14), there exists a unique weak solution for initial-boundary value problem (3). 
Inspired by [4], this paper will investigate the existence and uniqueness of weak solutions for problem (3) according to the following steps:

(i) First, we approximate nonlinear evolution problem (3) by nonlinear elliptic problems using an implicit iterative method (discretization in time-variable only), and then we prove the existence of a unique weak solution for each elliptic problem adopting a variational approach. These solutions constitute approximate solutions for problem (3).

(ii) Next, we show the uniqueness of solutions for initial-boundary value problem (3) using the monotonicity of the vector field $\mathbf{D}_{\nabla} \nabla .: u \in \mathbb{R}^{2} \longrightarrow \mathbf{D}_{\nabla u} \nabla u \in \mathbb{R}^{2}$.

(iii) Finally, passing to limits in some a priori energy estimates and using the monotonicity condition (17), we demonstrate the existence of weak solutions for problem (3).

\section{Preliminaries}

In this section, we state some useful lemmas that will be used later in the proofs.

Lemma 1. For all $a \geq 0$ and $b \geq 1$, we have $a b \leq a$ exp $(a)+b \log (b)$.

Proof. If $b \leq \exp (a)$, then $a b \leq a \exp (a) \leq a \exp (a)+b$ $\log (b)$.

If $\exp (a)<b$, then $a<\log (b)$, which means $a b<b \log (b)<a \exp (a)+b \log (b)$.

Lemma 2. Suppose $\phi: \mathbb{R}_{+} \longrightarrow \mathbb{R}_{+}$is a $C^{2}$ convex function. Then, for all $\xi_{0}, \xi_{1} \in \mathbb{R}^{2}$, we have

$$
\left(\mathbf{D}_{\xi_{1}} \xi_{1}-\mathbf{D}_{\xi_{0}} \xi_{0}\right) \cdot\left(\xi_{1}-\xi_{0}\right) \geq 0 \text {. }
$$

Proof. For each $t \in[0,1]$, we put $\xi_{t}=(1-t) \xi_{0}+t \xi_{1}$. Then, we have

$$
\begin{aligned}
\mathbf{D}_{\xi_{1}} \xi_{1}-\mathbf{D}_{\xi_{0}} \xi_{0}= & \int_{0}^{1} d\left[\left(\xi_{t} \cdot \mathbf{e}_{1}\right) g\left(\left|\xi_{t} \cdot \mathbf{e}_{1}\right|\right) \mathbf{e}_{1}\right]+\int_{0}^{1} d\left[\left(\xi_{t} \cdot \mathbf{e}_{2}\right) g\left(\left|\xi_{t} \cdot \mathbf{e}_{2}\right|\right) \mathbf{e}_{2}\right] \\
& +\int_{0}^{1} d\left[\left(\xi_{t} \cdot \mathbf{e}_{12}\right) g\left(\left|\xi_{t} \cdot \mathbf{e}_{12}\right|\right) \mathbf{e}_{12}\right]+\int_{0}^{1} d\left[\left(\xi_{t} \cdot \mathbf{e}_{-12}\right) g\left(\left|\xi_{t} \cdot \mathbf{e}_{-12}\right|\right) \mathbf{e}_{-12}\right]
\end{aligned}
$$

Since $\phi^{\prime \prime}(s)=g(s)+s g^{\prime}(s)$, then we obtain

$$
\begin{aligned}
\mathbf{D}_{\xi_{1}} \xi_{1}-\mathbf{D}_{\xi_{0}} \xi_{0}= & \int_{0}^{1}\left[\phi^{\prime \prime}\left(\left|\xi_{t} \cdot \mathbf{e}_{1}\right|\right)\left(\left(\xi_{1}-\xi_{0}\right) \cdot \mathbf{e}_{1}\right) \mathbf{e}_{1}\right] \mathrm{d} t+\int_{0}^{1}\left[\phi^{\prime \prime}\left(\left|\xi_{t} \cdot \mathbf{e}_{2}\right|\right)\left(\left(\xi_{1}-\xi_{0}\right) \cdot \mathbf{e}_{2}\right) \mathbf{e}_{2}\right] \mathrm{d} t \\
& +\int_{0}^{1}\left[\phi^{\prime \prime}\left(\left|\xi_{t} \cdot \mathbf{e}_{12}\right|\right)\left(\left(\xi_{1}-\xi_{0}\right) \cdot \mathbf{e}_{12}\right) \mathbf{e}_{12}\right] \mathrm{d} t+\int_{0}^{1}\left[\phi^{\prime \prime}\left(\left|\xi_{t} \cdot \mathbf{e}_{-12}\right|\right)\left(\left(\xi_{1}-\xi_{0}\right) \cdot \mathbf{e}_{-12}\right) \mathbf{e}_{-12}\right] \mathrm{d} t
\end{aligned}
$$

We conclude then

$$
\begin{aligned}
\left(\mathbf{D}_{\xi_{1}} \xi_{1}-\mathbf{D}_{\xi_{0}} \xi_{0}\right) \cdot\left(\xi_{1}-\xi_{0}\right)= & \int_{0}^{1}\left[\phi^{\prime \prime}\left(\left|\xi_{t} \cdot \mathbf{e}_{1}\right|\right)\left(\left(\xi_{1}-\xi_{0}\right) \cdot \mathbf{e}_{1}\right)^{2}\right] \mathrm{d} t+\int_{0}^{1}\left[\phi^{\prime \prime}\left(\left|\xi_{t} \cdot \mathbf{e}_{2}\right|\right)\left(\left(\xi_{1}-\xi_{0}\right) \cdot \mathbf{e}_{2}\right)^{2}\right] \mathrm{d} t \\
& +\int_{0}^{1}\left[\phi^{\prime \prime}\left(\left|\xi_{t} \cdot \mathbf{e}_{12}\right|\right)\left(\left(\xi_{1}-\xi_{0}\right) \cdot \mathbf{e}_{12}\right)^{2}\right] \mathrm{d} t \\
& +\int_{0}^{1}\left[\phi^{\prime \prime}\left(\left|\xi_{t} \cdot \mathbf{e}_{-12}\right|\right)\left(\left(\xi_{1}-\xi_{0}\right) \cdot \mathbf{e}_{-12}\right)^{2}\right] \mathrm{d} t \geq 0,
\end{aligned}
$$

which completes the proof.

Lemma 3. Uniform integrability and weak convergence [15].

Assume $\Omega \subset \mathbb{R}^{2}$ is bounded, and let $\left\{u_{i}\right\}_{i=1}^{\infty}$ be a sequence of functions in $L^{1}(\Omega)$ satisfying

$$
\sup _{i}\left\|u_{i}\right\|_{L^{1}(\Omega)}<\infty \text {. }
$$

Suppose also

$$
\lim _{l \longrightarrow \infty} \sup _{i} \int_{\Omega \cap\left\{\left|u_{i}\right| \geq l\right\}}\left|u_{i}\right| \mathrm{d} x=0 .
$$

Then, there exist a subsequence $\left\{u_{i_{j}}\right\}_{j=1}^{\infty}$ and $\tilde{u} \in L^{1}(\Omega)$ such that

$$
u_{i_{j}} \rightarrow \widetilde{u}, \quad \text { weakly in } L^{1}(\Omega) .
$$


Lemma 4. Assume $\Omega \subset \mathbb{R}^{2}$ is bounded, and let $\left\{u_{i}\right\}_{i=1}^{\infty}$ be a sequence of functions in $L^{1}(\Omega)$ such that

$$
\sup _{i} \int_{\Omega \cap\left\{\left|u_{i}\right| \geq k_{m}\right\}}\left|u_{i}\right| \log \left(\left|u_{i}\right|\right) \mathrm{d} x<\infty .
$$

Then, there exist a subsequence $\left\{u_{i_{j}}\right\}_{j=1}^{\infty}$ and $\tilde{u} \in L^{1}(\Omega)$ such that

$$
u_{i_{j}} \rightarrow \widetilde{u}, \quad \text { weakly in } L^{1}(\Omega),
$$

with $\tilde{u} \in L \log L^{k_{m}}(\Omega)$.

Proof. Given $M>0$, we may find an $l \geq k_{m}$ such that $M s \leq s \log (s)$ for all $s \geq l$. Consequently,

$$
\begin{aligned}
\int_{\Omega}\left|u_{i}\right| \mathrm{d} x & =\int_{\Omega \cap\left\{\left|u_{i}\right|<k_{m}\right\}}\left|u_{i}\right| \mathrm{d} x+\int_{\Omega \cap\left\{\left|u_{i}\right| \geq k_{m}\right\}}\left|u_{i}\right| \mathrm{d} x \\
& \leq k_{m}|\Omega|+\frac{1}{M} \int_{\Omega \cap\left\{\left|u_{i}\right| \geq k_{m}\right\}}\left|u_{i}\right| \log \left(\left|u_{i}\right|\right) \mid \mathrm{d} x,
\end{aligned}
$$

which implies that

$$
\sup _{i} \int_{\Omega}\left|u_{i}\right| \mathrm{d} x<\infty .
$$

On the other hand, there exists a positive constant $C$ such that

$$
\begin{aligned}
\int_{\Omega \cap\left\{\left|u_{i}\right| \geq l\right\}}\left|u_{i}\right| \mathrm{d} x & \leq \frac{1}{M} \int_{\Omega \cap\left\{\left|u_{i}\right| \geq l\right\}}\left|u_{i}\right| \log \left(\left|u_{i}\right|\right) \mathrm{d} x \\
& \leq \frac{1}{M} \int_{\Omega \cap\left\{\left|u_{i}\right| \geq k_{m}\right\}}\left|u_{i}\right| \log \left(\left|u_{i}\right|\right) \mathrm{d} x \\
& \leq \frac{C}{M}=\varepsilon,
\end{aligned}
$$

which is true for all $i$ and arbitrary $\varepsilon>0$. It follows then that

$$
\lim _{l \longrightarrow \infty} \sup _{i} \int_{\Omega \cap\left\{\left|u_{i}\right| \geq l\right\}}\left|u_{i}\right| \mathrm{d} x=0 .
$$
Then, from Lemma 3 , there exist a subsequence $\left\{u_{i_{j}}\right\}_{j=1}^{\infty}$
of $\left\{u_{i}\right\}_{i=1}^{\infty}$ and a function $\tilde{u} \in L^{1}(\Omega)$ such that

$$
u_{i_{j}} \rightarrow \widetilde{u}, \quad \text { weakly in } L^{1}(\Omega) .
$$

It remains to prove that $\tilde{u} \in L \log L^{k_{m}}(\Omega)$.

We know that the function $f(s)=s \log (s)$ for $s \geq 1$ is increasing and convex, and then the function $f(|s|)$ is also convex for all $s \geq 1$. Therefore, we obtain

$$
f(|\widetilde{u}|) \leq f\left(\left|u_{i_{j}}\right|\right)+f^{\prime}(|\widetilde{u}|)\left(\widetilde{u}-u_{i_{j}}\right) .
$$

Integrating the above inequality over $\Omega_{N} \cap\left\{\left|u_{i_{j}}\right| \geq k_{m}\right\}$ with $\Omega_{N}=\Omega \cap\left\{k_{m} \leq|\widetilde{u}| \leq N\right\}$, we have

$$
\begin{aligned}
\int_{\Omega_{N}} f(|\widetilde{u}|) \mathrm{d} x & \leq \int_{\Omega \cap\left\{\left|u_{i_{j}}\right| \geq k_{m}\right\}} f\left(\left|u_{i_{j}}\right|\right) \mathrm{d} x+\int_{\Omega_{N} \cap\left\{\left|u_{i_{j}}\right| \geq k_{m}\right\}} f^{\prime}(|\widetilde{u}|)\left(\tilde{u}-u_{i_{j}}\right) \mathrm{d} x \\
& \leq \int_{\Omega \cap\left\{\left|u_{i_{j}}\right| \geq k_{m}\right\}} f\left(\left|u_{i_{j}}\right|\right) \mathrm{d} x+\int_{\Omega \cap\left\{\left|u_{i_{j}}\right| \geq k_{m}\right\}} f^{\prime}(|\widetilde{u}|) \chi_{\left\{k_{m} \leq|\widetilde{u}| \leq N\right\}}\left(\widetilde{u}-u_{i_{j}}\right) \mathrm{d} x .
\end{aligned}
$$

Since $f^{\prime}(|\widetilde{u}|) \chi_{\left\{k_{m} \leq|\widetilde{u}| \leq N\right\}} \in L^{\infty}(\Omega)$ and passing to limits as $j \longrightarrow \infty$, we get

$$
\int_{\Omega_{N}} f(|\widetilde{u}|) \mathrm{d} x \leq \liminf _{j \rightarrow \infty} \int_{\Omega \cap\left\{\left|u_{i_{j}}\right| \geq k_{m}\right\}} f\left(\left|u_{i_{j}}\right|\right) \mathrm{d} x<\infty .
$$

Then, passing to limits as $N \longrightarrow \infty$, we deduce

$$
\int_{\Omega \cap\left\{\widetilde{u} \mid \geq k_{m}\right\}}|\widetilde{u}| \log (|\widetilde{u}|) \mathrm{d} x<\infty .
$$

It follows then $\tilde{u} \in L \log L^{k_{m}}(\Omega)$. This finishes the proof.

\section{Approximate Solutions}

In this section, we will discretize the time-variable interval $[0, T]$ to get approximate solutions for problem (3). We denote $h=(T / N)$ with $N \in \mathbb{N}^{*}$, and we designate by $u_{n}$ an approximate solution at time $n h$. We define gradually from $n=1,2, \ldots, N$ the following elliptic problems:

$$
\begin{cases}\frac{u_{n}-u_{n-1}}{h}-\nabla \cdot\left[\mathbf{D}_{\nabla u_{n}} \nabla u_{n}\right]=0, & \text { in } \Omega, \\ \left\langle\mathbf{D}_{\nabla u_{n}} \nabla u_{n}, \mathbf{n}\right\rangle=0, & \text { on } \partial \Omega .\end{cases}
$$

To solve these equations step by step, we only need to prove the existence and uniqueness of weak solutions of the following elliptic problems:

$$
\begin{cases}\frac{u-u_{0}}{h}-\nabla \cdot\left[\mathbf{D}_{\nabla u} \nabla u\right]=0, & \text { in } \Omega, \\ \left\langle\mathbf{D}_{\nabla u} \nabla u, \mathbf{n}\right\rangle=0, & \text { on } \partial \Omega,\end{cases}
$$

where $h>0$ and $u_{0} \in L^{2}(\Omega)$.

Definition 2. A function $u \in L^{2}(\Omega) \cap W^{1,1}(\Omega)$ with $\partial_{x_{i}} u \in L \log L^{k_{m}}(\Omega)$ for $i=1,2$ is called a weak solution for problem (36); if for any $\varphi \in C^{1}(\bar{\Omega})$, we have

$$
\int_{\Omega} \frac{u-u_{0}}{h} \varphi \mathrm{d} x+\int_{\Omega} \mathbf{D}_{\nabla u} \nabla u \cdot \nabla \varphi \mathrm{d} x=0 .
$$

And when $\varphi$ is a constant function, we obtain 


$$
\int_{\Omega} u \mathrm{~d} x=\int_{\Omega} u_{0} \mathrm{~d} x
$$

In order to prove the existence and uniqueness of weak solutions for problem (36), we consider the variational problem

$$
\min \{E(u) \mid u \in U\},
$$

where

$$
U=\left\{u \in L^{2}(\Omega) \cap W^{1,1}(\Omega) \mid \partial_{x_{i}} u \in L \log L^{k_{m}}(\Omega) \text { with } i=1,2, \int_{\Omega} u \mathrm{~d} x=\int_{\Omega} u_{0} \mathrm{~d} x\right\}
$$

and when $u \in U$, the functional $E$ is defined as

$$
E(u)=\int_{\Omega}\left[\phi\left(\left|u_{x_{1}}\right|\right)+\phi\left(\left|u_{x_{2}}\right|\right)+\phi\left(\left|u_{x_{12}}\right|\right)+\phi\left(\left|u_{x_{-12}}\right|\right)\right]+\frac{1}{2 h} \int_{\Omega}\left(u-u_{0}\right)^{2} d x .
$$

It is easy to prove that (36) is the Euler-Lagrange equations of the functional $E$ [16].

Theorem 2. Problem (36) has a unique weak solution.

Proof. Since

$$
0 \leq \inf _{u \in U} E(u) \leq E(0)=\frac{1}{2 h} \int_{\Omega} u_{0}^{2} \mathrm{~d} x,
$$

then we can construct a minimizing sequence $\left\{u_{q}\right\}_{q=1}^{\infty}$ in $U$ such that $E\left(u_{q}\right)<E(0)+1$ and

$$
\lim _{q \longrightarrow \infty} E\left(u_{q}\right)=\inf _{u \in U} E(u) .
$$

Besides,

$$
\begin{aligned}
& \int_{\Omega \cap\left\{\left|\partial_{x_{i}} u_{q}\right| \geq k_{m}\right\}}\left|\partial_{x_{i}} u_{q}\right| \log \left(\left|\partial_{x_{i}} u_{q}\right|\right) \mathrm{d} x \leq C \int_{\Omega \cap\left\{\left|\partial_{x_{i}} u_{q}\right| \geq k_{m}\right\}} \phi\left(\left|\partial_{x_{i}} u_{q}\right|\right) \mathrm{d} x \\
& \leq C E\left(u_{q}\right)<C(E(0)+1) \\
& \partial_{x_{i}} u_{q_{j}} \rightarrow \partial_{x_{i}} u_{1} \text {, weakly in } L^{1}(\Omega) \text {, } \\
& \partial_{x_{i}} u_{1} \in L \log L^{k_{m}}(\Omega) .
\end{aligned}
$$

with $C=\left(\varepsilon_{0}+\left(1 / A_{k_{m, 2}}\right)\right)>0$ and $i=1,2$. It follows then that for $i=1,2$,

$$
\sup _{q} \int_{\Omega \cap\left\{\left|\partial_{x_{i}} u_{q}\right| \geq k_{m}\right\}}\left|\partial_{x_{i}} u_{q}\right| \log \left(\left|\partial_{x_{i}} u_{q}\right|\right) \mathrm{d} x<\infty .
$$

Therefore, thanks to Lemma 4 and the weak compactness of $L^{2}(\Omega)$, we can find a subsequence $\left\{u_{q_{j}}\right\}_{j=1}^{\infty}$ of $\left\{u_{q}\right\}_{q=1}^{\infty}$ and a function $u_{1} \in L^{2}(\Omega) \cap W^{1,1}(\Omega)$ such that

$$
u_{q_{j}} \rightarrow u_{1} \text {, weakly in } L^{2}(\Omega) \text {, }
$$

Therefore, we have

$$
\begin{array}{r}
\int_{\Omega} u_{1} \mathrm{~d} x=\lim _{j \rightarrow \infty} \int_{\Omega} u_{q_{j}} \mathrm{~d} x=\int_{\Omega} u_{0} \mathrm{~d} x, \\
\int_{\Omega}\left(u_{1}-u_{0}\right)^{2} \mathrm{~d} x \leq \liminf _{j \rightarrow \infty} \int_{\Omega}\left(u_{q_{j}}-u_{0}\right)^{2} \mathrm{~d} x,
\end{array}
$$

and following the reasoning in the proof of Lemma 4 , it is easy to show that for any $a \in\left\{x_{1}, x_{2}, x_{12}, x_{-12}\right\}$ and for a fixed $\epsilon>0$, there exists $l \geq k_{m}$ such that

and for $i=1,2$, 


$$
\begin{gathered}
\int_{\Omega \cap\left\{\left|\partial_{a} u_{1}\right| \geq l\right\}}\left|\partial_{a} u_{1}\right| \log \left(\left|\partial_{a} u_{1}\right|\right) \mathrm{d} x \leq \liminf _{j \rightarrow \infty} \int_{\Omega \cap\left\{\left|\partial_{a} u_{q_{j}}\right| \geq l\right\}}\left|\partial_{a} u_{q_{j}}\right| \log \left(\left|\partial_{a} u_{q_{j}}\right|\right) \mathrm{d} x, \\
\int_{\Omega \cap\left\{\left|\partial_{a} u_{1}\right| \geq l\right\}} \phi\left(\left|\partial_{a} u_{1}\right|\right) \mathrm{d} x \leq\left(\varepsilon+A_{k_{m}, 2}\right)\left(\varepsilon+\frac{1}{A_{k_{m}, 2}}\right) \liminf \inf _{j \rightarrow \infty} \int_{\Omega \cap\left\{\left|\partial_{a} u_{q_{j}}\right| \geq l\right\}} \phi\left(\left|\partial_{a} u_{q_{j}}\right|\right) \mathrm{d} x .
\end{gathered}
$$

Similarly, since $\phi$ is increasing and convex in $[0, l]$, then we can prove that

$$
\int_{\Omega \cap\left\{\left|\partial_{a} u_{1}\right|<l\right\}} \phi\left(\left|\partial_{a} u_{1}\right|\right) \mathrm{d} x \leq \liminf _{j \rightarrow \infty} \int_{\Omega \cap\left\{\left|\partial_{a} u_{q_{j}}\right|<l\right\}} \phi\left(\left|\partial_{a} u_{q_{j}}\right|\right) \mathrm{d} x .
$$

Therefore, we obtain from (52) and (53) that

$$
\begin{aligned}
\int_{\Omega} \phi\left(\left|\partial_{a} u_{1}\right|\right) \mathrm{d} x & =\int_{\Omega \cap\left\{\left|\partial_{a} u_{1}\right|<l\right\}} \phi\left(\left|\partial_{a} u_{1}\right|\right) \mathrm{d} x+\int_{\Omega \cap\left\{\left|\partial_{a} u_{1}\right| \geq l\right\}} \phi\left(\left|\partial_{a} u_{1}\right|\right) \mathrm{d} x \\
& \leq\left(\varepsilon+A_{k_{m}, 2}\right)\left(\varepsilon+\frac{1}{A_{k_{m}, 2}}\right) \liminf _{j \rightarrow \infty} \int_{\Omega} \phi\left(\left|\partial_{a} u_{q_{j}}\right|\right) \mathrm{d} x
\end{aligned}
$$

Thus, by letting $\varepsilon \longrightarrow 0$, we get

$$
\int_{\Omega} \phi\left(\left|\partial_{a} u_{1}\right|\right) \mathrm{d} x \leq \liminf _{j \rightarrow \infty} \int_{\Omega} \phi\left(\left|\partial_{a} u_{q_{j}}\right|\right) \mathrm{d} x,
$$

for any $a \in\left\{x_{1}, x_{2}, x_{12}, x_{-12}\right\}$. It follows then that

$$
E\left(u_{1}\right) \leq \liminf _{j \rightarrow \infty} E\left(u_{q_{j}}\right)=\inf _{u \in U} E(u),
$$

which signifies that $u_{1} \in U$ is a minimizer of the energy functional $E(u)$, i.e.,

$$
E\left(u_{1}\right)=\min _{u \in U} E(u) .
$$

Furthermore, for all $\varphi \in C^{1}(\bar{\Omega})$ and $t \in \mathbb{R}$, we have $u_{1}+$ $t\left(\varphi-\varphi_{\Omega}\right) \in U$ with $\varphi_{\Omega}=(1 /|\Omega|) \int_{\Omega} \varphi \mathrm{d} x$. Then, $\rho(0) \leq \rho(t)$ where

$$
\rho(t)=E\left(u_{1}+t\left(\varphi-\varphi_{\Omega}\right)\right) .
$$

Hence, we have $\rho^{\prime}(0)=0$, which means

$$
\int_{\Omega} \frac{u_{1}-u_{0}}{h}\left(\varphi-\varphi_{\Omega}\right) \mathrm{d} x+\int_{\Omega} \mathbf{D}_{\nabla u_{1}} \nabla u_{1} \cdot \nabla \varphi \mathrm{d} x=0 .
$$

Because of (50), we get

$$
\int_{\Omega} \frac{u_{1}-u_{0}}{h} \varphi \mathrm{d} x+\int_{\Omega} \mathbf{D}_{\nabla u_{1}} \nabla u_{1} \cdot \nabla \varphi \mathrm{d} x=0 .
$$

We conclude then that $u_{1}$ is a weak solution for problem (36).

Now, assume that there is another weak solution $\widehat{u}$ of (36). Then, for every $\varphi \in C^{1}(\bar{\Omega})$, we have

$$
\int_{\Omega} \frac{\widehat{u}-u_{0}}{h} \varphi \mathrm{d} x+\int_{\Omega} \mathbf{D}_{\nabla \hat{u}} \nabla \widehat{u} \cdot \nabla \varphi \mathrm{d} x=0,
$$

which leads to

$$
\int_{\Omega} \frac{\widehat{u}-u_{1}}{h} \varphi \mathrm{d} x+\int_{\Omega}\left[\mathbf{D}_{\nabla \widehat{u}} \nabla \widehat{u}-\mathbf{D}_{\nabla u_{1}} \nabla u_{1}\right] \cdot \nabla \varphi \mathrm{d} x=0 .
$$

Then, if we choose $\varphi=\widehat{u}-u_{1}$ as a test function in (62), we get

$$
\int_{\Omega} \frac{\left(\widehat{u}-u_{1}\right)^{2}}{h} \mathrm{~d} x+\int_{\Omega}\left[\mathbf{D}_{\nabla \widehat{u}} \nabla \widehat{u}-\mathbf{D}_{\nabla u_{1}} \nabla u_{1}\right] \cdot\left(\nabla \widehat{u}-\nabla u_{1}\right) \mathrm{d} x=0 .
$$

Thanks to Lemma 2, we deduce that

$$
\int_{\Omega} \frac{\left(\widehat{u}-u_{1}\right)^{2}}{h} \mathrm{~d} x=0
$$


Therefore, $\widehat{u}=u_{1}$ a.e. in $\Omega$.

In conclusion, we have shown that there exists a unique weak solution $u_{n} \in U$ satisfying (35) for every $n \in\{1,2, \ldots, N\}$. Consequently, we define an approximate solution $u_{h}$ for problem (3) as

$$
u_{h}(x, t)= \begin{cases}u_{0}(x), & t=0, \\ u_{1}(x), & t \in(0, h], \\ \ldots \ldots \ldots, & \ldots \ldots, \\ u_{j}(x), & t \in((j-1) h, j h], \\ \ldots \ldots \ldots, & \ldots \ldots, \\ u_{N}(x), & t \in((N-1) h, T],\end{cases}
$$

for every $h=(T / N)$.

\section{Existence and Uniqueness of Weak Solutions}

Proof. of Theorem 1. In the beginning, we establish the uniqueness of solutions for problem (3). For this purpose, we suppose there exist two weak solutions $u$ and $v$ for problem (3). Then, we obtain the following:

$$
\begin{cases}\frac{\partial(u-v)}{\partial t}-\nabla \cdot\left[\mathbf{D}_{\nabla u} \nabla u-\mathbf{D}_{\nabla v} \nabla v\right]=0, & \text { in } Q_{T}, \\ \left\langle\mathbf{D}_{\nabla u} \nabla u-\mathbf{D}_{\nabla v} \nabla v, \mathbf{n}\right\rangle=0, & \text { on } \partial \Omega \times(0, T], \\ (u-v)(x ; 0)=0, & \text { in } \Omega .\end{cases}
$$

By multiplying the first equation of the above problem by $(u-v)$ and integrating over $\Omega$ and $[0, t]$, we get

$$
\frac{1}{2} \int_{\Omega}(u-v)^{2}(t) \mathrm{d} x+\int_{0}^{t} \int_{\Omega}\left[\mathbf{D}_{\nabla u} \nabla u-\mathbf{D}_{\nabla v} \nabla v\right] \cdot \nabla(u-v) \mathrm{d} x \mathrm{~d} \tau=0,
$$

for every $t \in(0, T]$. Since the second term of the above equation is nonnegative (thanks to Lemma 2), it follows then $u=v$ a.e. in $Q_{T}$.

Let us now find our weak solution for problem (3). We intend to send $h$ to zero and show that a subsequence of our solutions $u_{h}$ of the approximate problems (35) converges to a weak solution for problem (3). To this end, we need to find some a priori estimates.

It follows from (35) that for every $\varphi \in C^{1}(\bar{\Omega})$,

$$
\int_{\Omega} \frac{u_{n}-u_{n-1}}{h} \varphi \mathrm{d} x+\int_{\Omega} \mathbf{D}_{\nabla u_{n}} \nabla u_{n} \cdot \nabla \varphi \mathrm{d} x=0 .
$$

Then, by taking $u_{n}$ as a test function in (68) and using $u_{n} u_{n-1} \leq\left(\left(u_{n}^{2}+u_{n-1}^{2}\right) / 2\right)$, we get

$\frac{1}{2} \int_{\Omega} u_{n}^{2} \mathrm{~d} x+h \int_{\Omega} \mathbf{D}_{\nabla u_{n}} \nabla u_{n} \cdot \nabla u_{n} \mathrm{~d} x \leq \frac{1}{2} \int_{\Omega} u_{n-1}^{2} \mathrm{~d} x$.

For each $t \in(0, T]$, we can find $j \in\{1, \ldots, N\}$ such that $t \in((j-1) h, j h]$. Then, by adding all the inequalities (69) from $n=1$ to $n=j$, we get

$$
\frac{1}{2} \int_{\Omega} u_{j}^{2} \mathrm{~d} x+h \sum_{n=1}^{j} \int_{\Omega} \mathbf{D}_{\nabla u_{n}} \nabla u_{n} \cdot \nabla u_{n} \mathrm{~d} x \leq \frac{1}{2} \int_{\Omega} u_{0}^{2} \mathrm{~d} x .
$$
that

Then, by definition of $u_{h}$, we obtain for $t \in((j-1) h, j h]$

$$
\frac{1}{2} \int_{\Omega} u_{h}^{2}(x, t) \mathrm{d} x+\int_{0}^{j h} \int_{\Omega} \mathbf{D}_{\nabla u_{h}} \nabla u_{h} \cdot \nabla u_{h} \mathrm{~d} x \mathrm{~d} \tau \leq \frac{1}{2} \int_{\Omega} u_{0}^{2} \mathrm{~d} x .
$$

Since $\mathbf{D}_{\nabla u_{h}}$ is a symmetric positive definite matrix, we have also

$$
\frac{1}{2} \int_{\Omega} u_{h}^{2}(x, t) \mathrm{d} x+\int_{0}^{t} \int_{\Omega} \mathbf{D}_{\nabla u h} \nabla u_{h} \cdot \nabla u_{h} \mathrm{~d} x \mathrm{~d} \tau \leq \frac{1}{2} \int_{\Omega} u_{0}^{2} \mathrm{~d} x .
$$

Therefore, after taking the supremum over $[0, T]$, we deduce that

$$
\sup _{0 \leq t \leq T} \int_{\Omega} u_{h}^{2}(x, t) \mathrm{d} x+\int_{0}^{T} \int_{\Omega} \mathbf{D}_{\nabla u_{h}} \nabla u_{h} \cdot \nabla u_{h} \mathrm{~d} x \mathrm{~d} \tau \leq 2 \int_{\Omega} u_{0}^{2} \mathrm{~d} x .
$$

Recalling that $0 \leq \phi(s) \leq s \phi^{\prime}(s)$ for all $s \geq 0$, then we can derive the following:

$$
\begin{aligned}
\mathbf{D}_{\nabla u_{h}} \nabla u_{h} \cdot \nabla u_{h}= & \left|\nabla u_{h} \cdot \mathbf{e}_{1}\right| \phi^{\prime}\left(\left|\nabla u_{h} \cdot \mathbf{e}_{1}\right|\right)+\left|\nabla u_{h} \cdot \mathbf{e}_{2}\right| \phi^{\prime}\left(\left|\nabla u_{h} \cdot \mathbf{e}_{2}\right|\right) \\
& +\left|\nabla u_{h} \cdot \mathbf{e}_{12}\right| \phi^{\prime}\left(\left|\nabla u_{h} \cdot \mathbf{e}_{12}\right|\right)+\left|\nabla u_{h} \cdot \mathbf{e}_{-12}\right| \phi^{\prime}\left(\left|\nabla u_{h} \cdot \mathbf{e}_{-12}\right|\right) \\
\geq & \phi\left(\left|\nabla u_{h} \cdot \mathbf{e}_{1}\right|\right)+\phi\left(\left|\nabla u_{h} \cdot \mathbf{e}_{2}\right|\right)+\phi\left(\left|\nabla u_{h} \cdot \mathbf{e}_{12}\right|\right)+\phi\left(\left|\nabla u_{h} \cdot \mathbf{e}_{-12}\right|\right) .
\end{aligned}
$$


Besides, as in (46), for $\left|\partial_{x_{1}} u_{h}\right|,\left|\partial_{x_{2}} u_{h}\right| \geq k_{m}$, we may find a positive constant $C$ such that

$$
\begin{aligned}
\left|\partial_{x_{1}} u_{h}\right| \log \left(\left|\partial_{x_{1}} u_{h}\right|\right)+\left|\partial_{x_{2}} u_{h}\right| \log \left(\left|\partial_{x_{2}} u_{h}\right|\right) & \leq C\left(\phi\left(\left|\nabla u_{h} \cdot \mathbf{e}_{1}\right|\right)+\phi\left(\left|\nabla u_{h} \cdot \mathbf{e}_{2}\right|\right)\right) \\
& \leq C \mathbf{D}_{\nabla u_{h}} \nabla u_{h} \cdot \nabla u_{h} .
\end{aligned}
$$

Thus, we conclude

$$
\left\{\begin{array}{l}
\sup _{0 \leq t \leq T} \int_{\Omega} u_{h}^{2}(x, t) \mathrm{d} x<\infty, \\
\int_{0}^{T} \int_{\Omega \cap\left\{\left|\partial_{x_{1}} u_{h}\right| \geq k_{m}\right.}\left|\partial_{x_{1}} u_{h}\right| \log \left(\left|\partial_{x_{1}} u_{h}\right|\right) \mathrm{d} x \mathrm{~d} \tau<\infty \\
\int_{0}^{T} \int_{\Omega \cap\left\{\left|\partial_{x_{2}} u_{h}\right| \geq k_{m}\right\}}\left|\partial_{x_{2}} u_{h}\right| \log \left(\left|\partial_{x_{2}} u_{h}\right|\right) \mathrm{d} x \mathrm{~d} \tau<\infty
\end{array}\right.
$$

By Lemma 4, we can find a subsequence of $\left\{u_{h}\right\}$ (for simplicity, we also denote it by $u_{h}$ ) such that [17]

$$
\begin{gathered}
u_{h} \rightarrow u, \quad \text { weakly_ } * \text { in } L^{\infty}\left(0, T ; L^{2}(\Omega)\right), \\
u_{h} \rightarrow u, \quad \text { weakly in } L^{1}\left(0, T ; W^{1,1}(\Omega)\right),
\end{gathered}
$$

with

$$
\left\{\begin{array}{l}
\sup _{0 \leq t \leq T} \int_{\Omega} u^{2}(x, t) \mathrm{d} x<\infty, \\
\int_{0}^{T} \int_{\Omega \cap\left\{\left|\partial_{x_{1}} u\right| \geq k_{m}\right\}}\left|\partial_{x_{1}} u\right| \log \left(\left|\partial_{x_{1}} u\right|\right) \mathrm{d} x \mathrm{~d} \tau<\infty, \\
\int_{0}^{T} \int_{\Omega \cap\left\{\left|\partial_{x_{2}} u\right| \geq k_{m}\right\}}\left|\partial_{x_{2}} u\right| \log \left(\left|\partial_{x_{2}} u\right|\right) \mathrm{d} x \mathrm{~d} \tau<\infty .
\end{array}\right.
$$

So, it remains to prove that $u$ is just a weak solution for problem (3). Let us now denote $\xi_{h}=\mathbf{D}_{\nabla u_{h}} \nabla u_{h}$. We will show that $\xi_{h}$ is bounded in $\left[L^{2}\left(Q_{T}\right)\right]^{2}$, so we may find a subsequence of $\xi_{h}$ that converges weakly in $\left[L^{2}\left(Q_{T}\right)\right]^{2}$ to a particular vector-valued function. Then, we will prove that this vector-valued function is equal almost everywhere to $\mathbf{D}_{\nabla u} \nabla u$ in $Q_{T}$ through monotonicity condition (17).

From the expression of $\mathbf{D}_{\nabla u_{h}}$, we can derive the following:

$$
\begin{aligned}
\left|\xi_{h}\right|= & \mid \frac{\partial_{x_{1}} u_{h}}{\left|\partial_{x_{1}} u_{h}\right|} \phi^{\prime}\left(\left|\partial_{x_{1}} u_{h}\right|\right) \mathbf{e}_{1}+\frac{\partial_{x_{2}} u_{h}}{\left|\partial_{x_{2}} u_{h}\right|} \phi^{\prime}\left(\left|\partial_{x_{2}} u_{h}\right|\right) \mathbf{e}_{2} \\
& +\frac{\partial_{x_{12}} u_{h}}{\left|\partial_{x_{12}} u_{h}\right|} \phi^{\prime}\left(\left|\partial_{x_{12}} u_{h}\right|\right) \mathbf{e}_{12}+\frac{\partial_{x_{-12}} u_{h}}{\left|\partial_{x_{-12}} u_{h}\right|} \phi^{\prime}\left(\left|\partial_{x_{-12}} u_{h}\right|\right) \mathbf{e}_{-12} \mid \\
\leq & 4 \phi^{\prime}\left(\left|\partial_{x_{1}} u_{h}\right|+\left|\partial_{x_{2}} u_{h}\right|\right) .
\end{aligned}
$$

Given $\varepsilon_{1}, \varepsilon_{2}>0$, we may find $l_{1}=l_{2}=k_{m}$ such that

$$
\begin{aligned}
\phi^{\prime}(s) & \leq M \log (s), \\
s & \leq \varepsilon_{2} s \log (s),
\end{aligned}
$$

for all $s \geq k_{m}$ with $M=\left(\varepsilon_{1}+A_{k_{m}, 2}\right)$. Thus, we can distinguish two cases:

(i) If $\left|\partial_{x_{1}} u_{h}\right|+\left|\partial_{x_{2}} u_{h}\right|<k_{m}$ then $\left|\xi_{h}\right|^{2} \leq\left(4 \phi^{\prime}\left(k_{m}\right)\right)^{2}$.

(ii) If $\left|\partial_{x_{1}} u_{h}\right|+\left|\partial_{x_{2}} u_{h}\right| \geq k_{m}$ then

$$
\begin{gathered}
\left|\xi_{h}\right| \leq 4 M \log \left(\left|\partial_{x_{1}} u_{h}\right|+\left|\partial_{x_{2}} u_{h}\right|\right), \\
\left|\xi_{h}\right|^{2} \leq(4 M)^{2}\left(1+\varepsilon_{2} \log (2)\right)\left(\left|\partial_{x_{1}} u_{h}\right| \log \left(\left|\partial_{x_{1}} u_{h}\right|\right)+\left|\partial_{x_{2}} u_{h}\right| \log \left(\left|\partial_{x_{2}} u_{h}\right|\right)\right), \\
\left|\xi_{h}\right| \exp \left(\frac{\left|\xi_{h}\right|}{4 M}\right) \leq 4 M\left(1+\varepsilon_{2} \log (2)\right)\left(\left|\partial_{x_{1}} u_{h}\right| \log \left(\left|\partial_{x_{1}} u_{h}\right|\right)+\left|\partial_{x_{2}} u_{h}\right| \log \left(\left|\partial_{x_{2}} u_{h}\right|\right)\right) .
\end{gathered}
$$

Then, $\left\{\xi_{h}\right\}$ is bounded in $\left[L^{2}\left(Q_{T}\right)\right]^{2}$, which means that we can find a subsequence of $\left\{\xi_{h}\right\}$ (denote it also by $\left\{\xi_{h}\right\}$ ) and a function $\xi \in\left[L^{2}\left(Q_{T}\right)\right]^{2}$ such that

$$
\xi_{h} \rightarrow \xi, \quad \text { weakly in }\left[L^{2}\left(Q_{T}\right)\right]^{2} \text {. }
$$

Since $s \mapsto s \exp (s)(s \geq 0)$ is increasing and convex, then as in the proof of Lemma 4 , we deduce that

$$
\begin{gathered}
\int_{0}^{T} \int_{\Omega \cap\left\{\left|\partial_{x_{1}} u\right|+\left|\partial_{x_{2}} u\right| \geq k_{m}\right\}}|\xi| \exp \left(\frac{|\xi|}{4 M}\right) \mathrm{d} x \mathrm{~d} t \leq \\
\liminf _{h \longrightarrow 0} \int_{0}^{T} \int_{\Omega \cap\left\{\left|\partial_{x_{1}} u_{h}\right|+\left|\partial_{x_{2}} u_{h}\right| \geq k_{m}\right\}}\left|\xi_{h}\right| \exp \left(\frac{\left|\xi_{h}\right|}{4 M}\right) \mathrm{d} x \mathrm{~d} t<\infty .
\end{gathered}
$$

Then, by using Lemma 1, we get 


$$
\begin{aligned}
\int_{0}^{T} \int_{\Omega}|\xi \cdot \nabla u| \mathrm{d} x \mathrm{~d} t \leq & \int_{0}^{T} \int_{\Omega}|\xi||\nabla u| \mathrm{d} x \mathrm{~d} t \\
\leq & \int_{0}^{T} \int_{\Omega}|\xi|\left(\left|\partial_{x_{1}} u\right|+\left|\partial_{x_{2}} u\right|\right) \mathrm{d} x \mathrm{~d} t \\
\leq & k_{m} \int_{0}^{T} \int_{\Omega \cap\left\{\left|\partial_{x_{1}} u\right|+\left|\partial_{x_{2}} u\right|<k_{m}\right\}}|\xi| \mathrm{d} x \mathrm{~d} t \\
& +\int_{0}^{T} \int_{\Omega \cap\left\{\left|\partial_{x_{1}} u\right|+\left|\partial_{x_{2}} u\right| \geq k_{m}\right\}}|\xi| \exp \left(\frac{|\xi|}{4 M}\right) \mathrm{d} x \mathrm{~d} t \\
& +4 M\left(1+\varepsilon_{2}\right)\left[\int_{0}^{T} \int_{\Omega \cap\left\{\left|\partial_{x_{1}} u\right| \geq k_{m}\right\}}\left|\partial_{x_{1}} u\right| \log \left(\left|\partial_{x_{1}} u\right|\right) \mathrm{d} x \mathrm{~d} t\right. \\
& \left.+\int_{0}^{T} \int_{\Omega \cap\left\{\left|\partial_{x_{2}} u\right| \geq k_{m}\right\}}\left|\partial_{x_{2}} u\right| \log \left(\left|\partial_{x_{2}} u\right|\right) \mathrm{d} x \mathrm{~d} t\right]<\infty .
\end{aligned}
$$

It follows then $\xi \cdot \nabla u \in L^{1}\left(Q_{T}\right)$. Next, we will show that $\xi=\mathbf{D}_{\nabla u} \nabla u$ a.e. in $Q_{T}$.

For each $\varphi \in C^{1}\left(Q_{T}\right)$ with $\varphi(., T)=0$, we take $\varphi(x, n h)$ as a test function in (35):

$$
\int_{\Omega} \frac{u_{n}(x)-u_{n-1}(x)}{h} \varphi(x, n h) \mathrm{d} x+\int_{\Omega} \mathbf{D}_{\nabla u_{n}} \nabla u_{n} \cdot \nabla \varphi(x, n h) \mathrm{d} x=0,
$$

$$
-\frac{1}{h} \int_{\Omega} u_{0}(x) \varphi(x, 0) \mathrm{d} x+\sum_{n=0}^{N-1} \int_{\Omega} u_{n}(x) \frac{\varphi(x, n h)-\varphi(x,(n+1) h)}{h} \mathrm{~d} x
$$$$
+\sum_{n=1}^{N} \int_{\Omega} \mathbf{D}_{\nabla u_{n}} \nabla u_{n} \cdot \nabla \varphi(x, n h) \mathrm{d} x=0 .
$$

From the definition of $u_{h}(65)$, we have

with $n \in\{1,2, \ldots, N\}$. By summing $n$ from 1 to $N$, we obtain

$$
\begin{aligned}
\sum_{n=0}^{N-1} \int_{\Omega} u_{n}(x) \frac{\varphi(x, n h)-\varphi(x,(n+1) h)}{h} \mathrm{~d} x & =-\sum_{n=0}^{N-1} \int_{n h}^{(n+1) h} \int_{\Omega} u_{h}(x, t) \frac{\varphi_{t}(x, t)}{h} \mathrm{~d} x \mathrm{~d} t \\
& =-\frac{1}{h} \int_{0}^{T} \int_{\Omega} u_{h}(x, t) \varphi_{t}(x, t) \mathrm{d} x \mathrm{~d} t .
\end{aligned}
$$

Therefore,

$$
\begin{aligned}
& -\int_{\Omega} u_{0}(x) \varphi(x, 0) \mathrm{d} x-\int_{0}^{T} \int_{\Omega} u_{h}(x, t) \varphi_{t}(x, t) \mathrm{d} x \mathrm{~d} t+\int_{0}^{T} \int_{\Omega} D_{\nabla u_{h}} \nabla u_{h} \cdot \nabla \varphi \mathrm{d} x \mathrm{~d} t \\
& +\sum_{n=1}^{N} \int_{(n-1) h}^{n h} \int_{\Omega} \mathbf{D}_{\nabla u_{n}} \nabla u_{n} \cdot[\nabla \varphi(x, n h)-\nabla \varphi(x, t)] \mathrm{d} x \mathrm{~d} t=0 .
\end{aligned}
$$

Letting $h$ tend to zero, we get

$$
\int_{\Omega} u_{0}(x) \varphi(x, 0) \mathrm{d} x+\int_{0}^{T} \int_{\Omega} u \varphi_{t} \mathrm{~d} x \mathrm{~d} t=\int_{0}^{T} \int_{\Omega} \xi \cdot \nabla \varphi \mathrm{d} x \mathrm{~d} t
$$


On the other hand, we let $v \in L^{1}\left(Q_{T}\right)$ with

$$
\int_{0}^{T} \int_{\Omega \cap\left\{\left|\partial_{x_{i}} v\right| \geq k_{m}\right\}}\left|\partial_{x_{i}} v\right| \log \left(\left|\partial_{x_{i}} v\right|\right) \mathrm{d} x \mathrm{~d} t<\infty,
$$

for $i=1,2$. We sum up inequalities (69):

$$
\frac{1}{2} \int_{\Omega} u_{h}^{2}(T) \mathrm{d} x+\int_{0}^{T} \int_{\Omega} \mathbf{D}_{\nabla u_{h}} \nabla u_{h} \cdot \nabla u_{h} \mathrm{~d} x \mathrm{~d} t \leq \frac{1}{2} \int_{\Omega} u_{0}^{2} \mathrm{~d} x .
$$

We have from Lemma 2 that

$$
\int_{0}^{T} \int_{\Omega}\left(\mathbf{D}_{\nabla u_{h}} \nabla u_{h}-\mathbf{D}_{\nabla v} \nabla v\right) \cdot\left(\nabla u_{h}-\nabla v\right) \mathrm{d} x \mathrm{~d} t \geq 0
$$

Then, we obtain

$$
\begin{aligned}
& \frac{1}{2} \int_{\Omega} u_{h}^{2}(T) \mathrm{d} x+\int_{0}^{T} \int_{\Omega} \mathbf{D}_{\nabla u h} \nabla u_{h} \cdot \nabla v \mathrm{~d} x \mathrm{~d} t+\int_{0}^{T} \int_{\Omega} \mathbf{D}_{\nabla v} \nabla v \cdot \nabla u_{h} \mathrm{~d} x \mathrm{~d} t \\
& \quad-\int_{0}^{T} \int_{\Omega} \mathbf{D}_{\nabla v} \nabla v \cdot \nabla v \mathrm{~d} x \mathrm{~d} t \leq \frac{1}{2} \int_{\Omega} u_{0}^{2} \mathrm{~d} x .
\end{aligned}
$$

Letting $h \longrightarrow 0$ and noting that

$$
\int_{\Omega} u^{2}(T) \mathrm{d} x \leq \liminf \operatorname{in}_{h \longrightarrow 0} \int_{\Omega} u_{h}^{2}(T) \mathrm{d} x
$$

$$
\begin{aligned}
& \frac{1}{2} \int_{\Omega} u^{2}(T) \mathrm{d} x+\int_{0}^{T} \int_{\Omega} \xi \cdot \nabla v \mathrm{~d} x \mathrm{~d} t+\int_{0}^{T} \int_{\Omega} \mathbf{D}_{\nabla v} \nabla v \cdot \nabla u \mathrm{~d} x \mathrm{~d} t \\
& \quad-\int_{0}^{T} \int_{\Omega} \mathbf{D}_{\nabla v} \nabla v \cdot \nabla v \mathrm{~d} x \mathrm{~d} t \leq \frac{1}{2} \int_{\Omega} u_{0}^{2} \mathrm{~d} x .
\end{aligned}
$$

By using $\varphi=u$ in (89), we get

$\frac{1}{2} \int_{\Omega} u^{2}(T) \mathrm{d} x+\frac{1}{2} \int_{\Omega} u_{0}^{2} \mathrm{~d} x=\int_{0}^{T} \int_{\Omega} \xi \cdot \nabla u \mathrm{~d} x \mathrm{~d} t$.

Combining (95) with (96), we have

$\int_{0}^{T} \int_{\Omega}\left(\xi-\mathbf{D}_{\nabla v} \nabla v\right) \cdot(\nabla v-\nabla u) \mathrm{d} x \mathrm{~d} t \leq-\int_{\Omega} u^{2}(T) \mathrm{d} x$

Now, setting $v=u+\lambda w$ for any $\lambda>0, w \in W^{1,2}\left(Q_{T}\right)$, we derive from the above inequality that

$$
\int_{0}^{T} \int_{\Omega}\left(\xi-\mathbf{D}_{\nabla(u+\lambda w)} \nabla(u+\lambda w)\right) \cdot \nabla w \mathrm{~d} x \mathrm{~d} t \leq 0 .
$$

By letting $\lambda \longrightarrow 0$ and using Lebesgue's dominated convergence theorem, we obtain

$$
\int_{0}^{T} \int_{\Omega}\left(\xi-\mathbf{D}_{\nabla u} \nabla u\right) \cdot \psi \mathrm{d} x \mathrm{~d} t=0,
$$

for every $\psi \in\left[L^{2}(\Omega)\right]^{2}$. It follows then

$$
\xi=\mathbf{D}_{\nabla u} \nabla u, \quad \text { a.e. in } Q_{T} .
$$

Therefore, we conclude from (89) that

$-\int_{\Omega} u_{0}(x) \varphi(x, 0) \mathrm{d} x+\int_{0}^{T} \int_{\Omega}\left[-u \varphi_{t}+\mathbf{D}_{\nabla u} \nabla u \cdot \nabla \varphi\right] \mathrm{d} x \mathrm{~d} t=0$,

for any $\varphi \in C^{1}\left(\bar{Q}_{T}\right)$ with $\varphi(., T)=0$. Finally, we need to prove that $u \in C\left([0, T], L^{2}(\Omega)\right)$. If we choose $\varphi \in C_{0}^{\infty}\left(Q_{T}\right)$ in (89), we obtain

$$
\int_{0}^{T} \int_{\Omega} u \varphi_{t} \mathrm{~d} x \mathrm{~d} t=\int_{0}^{T} \int_{\Omega} \xi \cdot \nabla \varphi \mathrm{d} x \mathrm{~d} t .
$$

Since $\xi \in\left[L^{2}\left(Q_{T}\right)\right]^{2}$, we conclude that $u_{t} \in L^{1}\left(0, T ; H^{-1}(\Omega)\right)$ where $H^{-1}(\Omega)$ is the dual space of $W_{0}^{1,2}(\Omega)$. Since

$$
\begin{gathered}
u=\int_{0}^{t} u_{\tau} \mathrm{d} \tau+u_{0}, \\
u_{0} \in L^{2}(\Omega) \hookrightarrow H^{-1}(\Omega) .
\end{gathered}
$$

It follows then that $u \in C\left(0, T ; H^{-1}(\Omega)\right)$. Besides, for every $h>0$, let $v_{h}(x, t)=u(x, t+h)$ be the weak solution for problem (3) satisfying $v_{h}(x ; 0)=u(x, h)$. Then, $w_{h}(x, t)=$ $u(x, t+h)-u(x, t)$ satisfies 


$$
\begin{cases}\frac{\partial w_{h}}{\partial t}-\nabla \cdot\left[\mathbf{D}_{\nabla v_{h}} \nabla v_{h}-\mathbf{D}_{\nabla u} \nabla u\right]=0, & \text { in } \Omega \times(0, T], \\ \left\langle\mathbf{D}_{\nabla v_{h}} \nabla v_{h}-\mathbf{D}_{\nabla u} \nabla u, \mathbf{n}\right\rangle=0, & \text { on } \partial \Omega \times(0, T], \\ w_{h}(x ; 0)=u(x, h)-u_{0}(x), & \text { in } \Omega .\end{cases}
$$

For each $t_{0} \in[0, T]$, we may choose $w_{h}$ as a test function in the first equation for problem (104) over $\left[0, t_{0}\right]$ :

$$
\frac{1}{2} \int_{\Omega} w_{h}^{2}\left(x, t_{0}\right) \mathrm{d} x+\int_{0}^{t_{0}} \int_{\Omega}\left(\mathbf{D}_{\nabla v_{h}} \nabla v_{h}-\mathbf{D}_{\nabla u} \nabla u\right) \cdot\left(\nabla v_{h}-\nabla u\right) \mathrm{d} x \mathrm{~d} t \leq \frac{1}{2} \int_{\Omega} w_{h}^{2}(x, 0) \mathrm{d} x
$$

Because of Lemma 2, we deduce

$$
\int_{\Omega}\left|u\left(x, t_{0}+h\right)-u\left(x, t_{0}\right)\right|^{2} \mathrm{~d} x \leq \int_{\Omega}\left|u(x, h)-u_{0}(x)\right|^{2} \mathrm{~d} x .
$$

Now, in order to prove that $u \in C\left([0, T], L^{2}(\Omega)\right)$, we need to prove

$$
\lim \sup _{h \longrightarrow 0^{+}} \int_{\Omega}\left|u(x, h)-u_{0}(x)\right|^{2} \mathrm{~d} x=0 .
$$

We suppose that (107) is not true. Then, there exist a positive number $\delta$ and a sequence $\left\{h_{i}\right\}$ with $h_{i} \longrightarrow 0$ as $i \longrightarrow \infty$ such that

$$
\lim _{h_{i} \longrightarrow 0^{+}} \int_{\Omega}\left|u\left(x, h_{i}\right)-u_{0}(x)\right|^{2} \mathrm{~d} x \geq \delta .
$$

From estimate (72), we have

$$
\int_{\Omega}\left|u\left(x, h_{i}\right)\right|^{2} \mathrm{~d} x \leq \int_{\Omega}\left|u_{0}(x)\right|^{2} \mathrm{~d} x .
$$

Then, from (108), we get

$$
\liminf _{h_{i} \longrightarrow 0^{+}}\left(\int_{\Omega}\left|u_{0}(x)\right|^{2} \mathrm{~d} x-\int_{\Omega} u_{0}(x) u\left(x, h_{i}\right) \mathrm{d} x\right) \geq \frac{\delta}{2} .
$$

From (109), we conclude that $\left\{u\left(x, h_{i}\right)\right\}$ is a bounded sequence in $L^{2}(\Omega)$. Then, we may find a subsequence (denote it also by $\left\{u\left(x, h_{i}\right)\right\}$ ) such that there exists a function $\tilde{u}_{0} \in L^{2}(\Omega)$ such that

$$
u\left(x, h_{i}\right) \rightarrow \widetilde{u}_{0}, \quad \text { weakly in } L^{2}(\Omega) .
$$

Since $u \in C\left(0, T ; H^{-1}(\Omega)\right)$, it follows that

$$
u\left(x, h_{i}\right) \rightarrow u_{0}, \quad \text { weakly in } H^{-1}(\Omega) .
$$

Therefore, we must have $\tilde{u}_{0}=u_{0}$, and since $u \in C\left(0, T ; H^{-1}(\Omega)\right)$, it follows that

$$
u\left(x, h_{i}\right) \rightarrow u_{0}, \quad \text { weakly in } L^{2}(\Omega),
$$

which is contradictory with (110). Therefore, we conclude that (107) is true and $u \in C\left([0, T], L^{2}(\Omega)\right)$. This completes the proof of Theorem 1 .

\section{Numerical Implementation and Experimental Results}

5.1. Consistent and Stable Symmetric Finite Difference Approximation. In this section, we provide a consistent and stable discretization scheme using symmetric finite difference approximation: at time $t_{n}=n \delta_{t}, n \geq 0$, and the mesh points $x_{i}=i \delta, y_{j}=j \delta(0 \leq i \leq N+1$ and $0 \leq j \leq M+1)$, and we denote by $u_{i, j}^{n}$ the finite difference approximation of $u\left(x_{i}, y_{j} ; t_{n}\right)$. The time-space derivatives are discretized in the following manner: 


$$
\begin{aligned}
& u_{x_{1}}\left(x_{i}, y_{j} ; t_{n}\right)=\frac{u\left(x_{i+(1 / 2)}, y_{j} ; t_{n}\right)-u\left(x_{i-(1 / 2)}, y_{j} ; t_{n}\right)}{\delta}+\mathcal{O}\left(\delta^{2}\right) \\
& u_{x_{2}}\left(x_{i}, y_{j} ; t_{n}\right)=\frac{u\left(x_{i}, y_{j+(1 / 2)} ; t_{n}\right)-u\left(x_{i}, y_{j-(1 / 2)} ; t_{n}\right)}{\delta}+\mathcal{O}\left(\delta^{2}\right) \text {, } \\
& u_{x_{12}}\left(x_{i}, y_{j} ; t_{n}\right)=\frac{u\left(x_{i+(1 / 2)}, y_{j+(1 / 2)} ; t_{n}\right)-u\left(x_{i-(1 / 2)}, y_{j-(1 / 2)} ; t_{n}\right)}{\sqrt{2} \delta}+\mathcal{O}\left(\delta^{2}\right) \text {, } \\
& u_{x_{-12}}\left(x_{i}, y_{j} ; t_{n}\right)=\frac{u\left(x_{i-(1 / 2)}, y_{j+(1 / 2)} ; t_{n}\right)-u\left(x_{i+(1 / 2)}, y_{j-(1 / 2)} ; t_{n}\right)}{\sqrt{2} \delta}+\mathcal{O}\left(\delta^{2}\right) \text {, } \\
& u_{t}\left(x_{i}, y_{j} ; t_{n}\right)=\frac{u\left(x_{i}, y_{j} ; t_{n+1}\right)-u\left(x_{i}, y_{j} ; t_{n}\right)}{\delta_{t}}+\mathcal{O}\left(\delta_{t}\right)
\end{aligned}
$$

By assume $\delta=1$ and denote

$$
\left\{\begin{array}{l}
g_{\mathbf{N}_{i, j}}^{n}=g\left(\left|\Delta_{\mathbf{N}} u_{i, j}^{n}\right|\right), \\
g_{\mathbf{E}_{i, j}}^{n}=g\left(\left|\Delta_{\mathbf{E}} u_{i, j}^{n}\right|\right), \\
g_{\mathbf{S}_{i, j}}^{n}=g\left(\left|\Delta_{\mathbf{S}} u_{i, j}^{n}\right|\right), \\
g_{\mathbf{W}_{i, j}}^{n}=g\left(\left|\Delta_{\mathbf{W}} u_{i, j}^{n}\right|\right), \\
g_{\mathbf{N E}_{i, j}}^{n}=g\left(\left|\frac{\Delta_{\mathbf{N E}} u_{i, j}^{n}}{\sqrt{2}}\right|\right), \\
g_{\mathbf{S E}_{i, j}}^{n}=g\left(\left|\frac{\Delta_{\mathbf{S E}} u_{i, j}^{n}}{\sqrt{2}}\right|\right), \\
g_{\mathbf{N W}_{i, j}}^{n}=g\left(\left|\frac{\Delta_{\mathbf{N W}} u_{i, j}^{n}}{\sqrt{2}}\right|\right), \\
g_{\mathbf{S W}_{i, j}}^{n}=g\left(\left|\frac{\Delta_{\mathbf{S W}} u_{i, j}^{n}}{\sqrt{2}}\right|\right), \\
\Delta_{\mathbf{E}} u_{i, j}^{n}=u_{i+1, j}^{n}-u_{i, j}^{n}, \\
\Delta_{\mathbf{S}} u_{i, j}^{n}=u_{i, j-1}^{n}-u_{i, j}^{n}, \\
\Delta_{\mathbf{W}} u_{i, j}^{n}=u_{i-1, j}^{n}-u_{i, j}^{n}, \\
\Delta_{\mathbf{N E}} u_{i, j}^{n}=u_{i+1, j+1}^{n}-u_{i, j}^{n}, \\
\Delta_{\mathbf{S E}} u_{i, j}^{n}=u_{i+1, j-1}^{n}-u_{i, j}^{n}, \\
\Delta_{\mathbf{S W}} u_{i, j}^{n}=u_{i-1, j-1}^{n}-u_{i, j}^{n}, \\
\Delta_{\mathbf{N W}} u_{i, j}^{n}=u_{i-1, j+1}^{n}-u_{i, j}^{n} .
\end{array}\right.
$$

Then, we may approximate problem (3) using the above scheme to obtain the following nonlinear diffusion filter:

$$
u_{i, j}^{n+1}=u_{i, j}^{n}+\delta_{t}\left[g_{\mathrm{N}} \Delta_{\mathrm{N}} u+g_{\mathrm{E}} \Delta_{\mathrm{E}} u+g_{\mathrm{S}} \Delta_{\mathrm{S}} u+g_{\mathrm{W}} \Delta_{\mathrm{W}} u+\frac{g_{\mathrm{NE}} \Delta_{\mathrm{NE}} u+g_{\mathrm{SE}} \Delta_{\mathrm{SE}} u+g_{\mathrm{SW}} \Delta_{\mathrm{SW}} u+g_{\mathrm{NW}} \Delta_{\mathrm{NW}} u}{2}\right]_{i, j}^{n}
$$




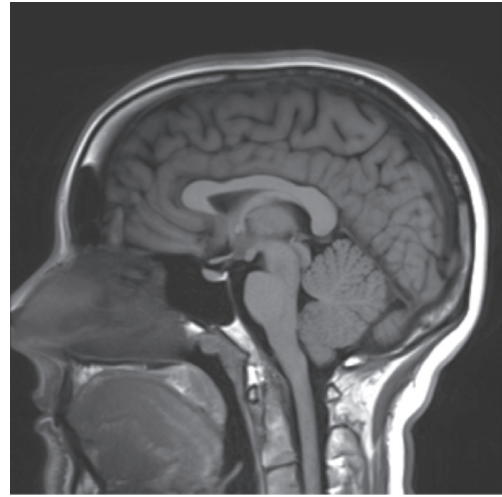

(a)

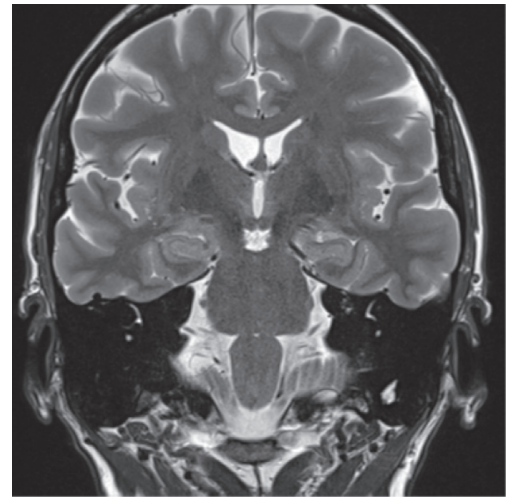

(b)

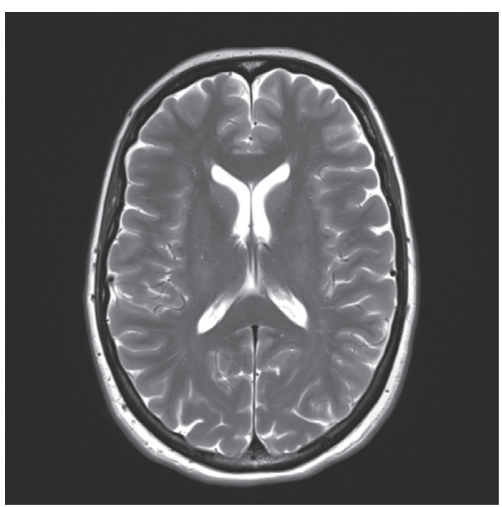

(c)

Figure 1: Brain MRI scans: Patient30: sagittal T1 of a 30-year-old female patient [20]. Patient50: coronal T2 of a 50-year-old female patient [21]. Patient55: axial T2 of a 55-year-old patient [22]. (a) Patient30, (b) Patient50, and (c) Patient55.

for $1 \leq i \leq \mathbf{N}, 1 \leq j \leq 1 \leq i \leq \mathbf{M}$, and $n \geq 0$, with the initial condition $u_{i, j}^{0}$ and the discrete Neumann boundary condition:

$$
\left\{\begin{array}{l}
u_{0, j}^{n}=u_{1, j}^{n}, u_{\mathbf{N}+1, j}^{n}=u_{\mathbf{N}, j}^{n}, \quad \text { for } 1 \leq j \leq \mathbf{M}, \\
u_{i, 0}^{n}=u_{i, 1}^{n}, u_{i, \mathbf{M}+1}^{n}=u_{i, \mathbf{M}}^{n}, \quad \text { for } 1 \leq i \leq \mathbf{N}, \\
u_{0,0}^{n}=u_{1,1}^{n}, u_{\mathbf{N}+1,0}^{n}=u_{\mathbf{N}, 1}^{n}, \\
u_{0, \mathbf{M}+1}^{n}=u_{1, \mathbf{M}}^{n}, u_{\mathbf{N}+1, \mathbf{M}+1}^{n}=u_{\mathbf{N}, \mathbf{M}}^{n} .
\end{array}\right.
$$

A unique sequence $\left(u^{n}\right)_{n \in \mathbb{N}}$ is produced when using filter (116) on a particular initial image $u^{0}$ [2]. Besides, due to the continuity of the function $g$, the sequence $u^{n}$ depends continuously on $u^{0}$ for every finite $n$. Furthermore, equation (116) satisfies the following maximum-minimum principle, which describes a stability condition for the discrete scheme.

Theorem 3. Discrete extremum principle [1, 2].

For an iteration step satisfying

$$
0<\delta_{t}<\frac{1}{6 g(0)}
$$

scheme (116) satisfies

$$
\min _{i, j} u_{i, j}^{0} \leq u_{i, j}^{n} \leq \max _{i, j} u_{i, j}^{0},
$$

for all $1 \leq i \leq N, 1 \leq j \leq M$, and $n \in \mathbb{N}$.

5.2. Experimental Results. This section will show the performance of proposed diffusion filter (116) in the image denoising process, under the boundary and initial conditions (117) while respecting the requirements concerning $\phi$ (Section 1), and $\delta_{t}$ (118). We will use the Peak Signal-toNoise Ratio (PSNR that is a positive value) [18] and the Structural SIMilarity Index (SSIM that lies in $(0,1)$ ) [19] to evaluate the quality of the restored images. The best results for the denoising process are equivalent to the higher value of these metrics.

For comparative purposes, we will examine the proposed diffusion function with another one that has the same properties using the same filter (116). Therefore, we will use the following diffusion functions:

(i) The proposed diffusion function $(m=1$ for instance):

$$
g(s)= \begin{cases}p_{0} P_{1,0 k}(s)+v_{0} P_{2,0 k}(s)+p_{k} P_{1, k 0}(s)+v_{k} P_{2, k 0}(s), & s \in[0, k[ \\ p_{k} g_{k, 1}(s)+v_{k} g_{k, 2}(s), & s \in[k, \infty[\end{cases}
$$

(ii) The Wang and Zhou diffusion function (WZ) [4]:

$$
g(s)=\frac{1}{s+1}+\frac{\log (s+1)}{s} .
$$

Additionally, we will consider real test images Figure 1 and evaluate our model's performance on these images, which will be corrupted with different levels of Gaussian white noises with zero mean and variance $\sigma^{2}$.

Table 1 shows the quantitative results on real images, corrupted with various Gaussian noises, filtered by discrete model (116) using proposed diffusion function (120) and the one proposed by WZ (121). These results are obtained using the optimal parameters determined experimentally, as in Table 2 for each diffusion function.

It can be seen from Table 1 and Figure 2 that the proposed model shows remarkable results against the $\mathrm{WZ}$ model. From a visual comparison, Figure 2 shows that the restored images using the proposed diffusion function have considerable noise removal and preserve the image essential features better than the restored images by the WZ diffusion function. Besides, compared with the $\mathrm{WZ}$ diffusion function, 
TABLE 1: PSNR and SSIM values of the images in Figure 1 affected by different values of Gaussian noise $\sigma^{2}$ and their corresponding iteration number for both functions.

\begin{tabular}{|c|c|c|c|c|c|c|c|c|c|}
\hline & \multirow[b]{2}{*}{$\sigma^{2}$} & \multicolumn{2}{|c|}{ Noisy } & \multicolumn{3}{|c|}{$\mathrm{WZ}$ [4] } & \multicolumn{3}{|c|}{ Proposed } \\
\hline & & PSNR & SSIM & PSNR & SSIM & Iter & PSNR & SSIM & Iter \\
\hline \multirow{5}{*}{ Patient30 } & 0.005 & 23.4311 & 0.3426 & 33.3757 & 0.9114 & 30 & 33.8333 & 0.9372 & 13 \\
\hline & 0.010 & 20.6839 & 0.2432 & 31.3367 & 0.8766 & 41 & 31.5737 & 0.9145 & 21 \\
\hline & 0.015 & 19.1190 & 0.1960 & 30.1565 & 0.8521 & 48 & 30.2008 & 0.8980 & 24 \\
\hline & 0.020 & 17.9763 & 0.1660 & 29.0905 & 0.8298 & 56 & 29.0007 & 0.8825 & 26 \\
\hline & 0.100 & 12.0756 & 0.0596 & 22.6821 & 0.6535 & 117 & 21.8953 & 0.7389 & 56 \\
\hline \multirow{5}{*}{ Patient50 } & 0.005 & 23.6686 & 0.4424 & 31.1185 & 0.8708 & 24 & 31.2934 & 0.8769 & 11 \\
\hline & 0.010 & 20.7561 & 0.3278 & 29.0893 & 0.8186 & 35 & 29.1527 & 0.8258 & 19 \\
\hline & 0.015 & 19.1313 & 0.2707 & 27.9137 & 0.7820 & 41 & 27.8981 & 0.7920 & 26 \\
\hline & 0.020 & 17.9838 & 0.2334 & 27.0011 & 0.7508 & 47 & 26.9714 & 0.7625 & 27 \\
\hline & 0.100 & 11.9985 & 0.0911 & 21.4469 & 0.5585 & 93 & 20.9674 & 0.5932 & 62 \\
\hline \multirow{5}{*}{ Patient55 } & 0.005 & 24.0179 & 0.3867 & 31.3190 & 0.9021 & 26 & 31.4668 & 0.9258 & 19 \\
\hline & 0.010 & 21.2303 & 0.2892 & 29.1310 & 0.8600 & 36 & 29.0997 & 0.8887 & 28 \\
\hline & 0.015 & 19.5990 & 0.2403 & 27.6640 & 0.8221 & 44 & 27.5635 & 0.8561 & 35 \\
\hline & 0.020 & 18.4292 & 0.2096 & 26.7717 & 0.7938 & 49 & 26.5468 & 0.8305 & 42 \\
\hline & 0.100 & 12.2234 & 0.0882 & 20.6948 & 0.5642 & 98 & 20.1755 & 0.6237 & 99 \\
\hline
\end{tabular}

TABLe 2: The best possible parameters for different diffusion functions.

\begin{tabular}{|c|c|c|c|c|c|c|c|c|}
\hline & & WZ [4] & & & & sed & & \\
\hline & $\sigma^{2}$ & $\delta_{t}$ & $\delta_{t}$ & $k$ & $p_{0}$ & $p_{k}$ & $v_{0}$ & $v_{k}$ \\
\hline & 0.005 & 0.08331 & 0.14701 & 4.61411 & 1.13191 & 0.66151 & -0.00011 & -0.10441 \\
\hline & 0.010 & 0.08331 & 0.14991 & 5.00191 & 1.10891 & 0.45921 & -0.00011 & -0.04351 \\
\hline Patient 30 & 0.015 & 0.08331 & 0.15051 & 5.20221 & 1.10601 & 0.45671 & -0.00021 & -0.03851 \\
\hline & 0.020 & 0.08331 & 0.14701 & 5.86411 & 1.13281 & 0.46991 & -0.00021 & -0.03961 \\
\hline & 0.100 & 0.08331 & 0.15001 & 5.08081 & 1.10111 & 0.45791 & -0.00091 & -0.03951 \\
\hline & 0.005 & 0.08331 & 0.14941 & 3.18011 & 1.09241 & 0.56271 & -0.00021 & -0.01151 \\
\hline & 0.010 & 0.08321 & 0.16231 & 1.89931 & 1.00171 & 0.56991 & -0.00001 & -0.00201 \\
\hline Patient50 & 0.015 & 0.08331 & 0.16591 & 2.06951 & 0.99011 & 0.56981 & -0.00011 & -0.11471 \\
\hline & 0.020 & 0.08331 & 0.16101 & 1.79891 & 1.00001 & 0.56981 & -0.00011 & -0.01051 \\
\hline & 0.100 & 0.08331 & 0.15301 & 3.87001 & 0.98701 & 0.55511 & -0.00031 & -0.13411 \\
\hline & 0.005 & 0.08321 & 0.14681 & 3.50081 & 1.12891 & 0.58971 & -0.00021 & -0.16571 \\
\hline & 0.010 & 0.08331 & 0.14701 & 3.31431 & 1.12621 & 0.58281 & -0.00131 & -0.17311 \\
\hline Patient55 & 0.015 & 0.08331 & 0.15141 & 3.09991 & 1.09021 & 0.58881 & -0.01401 & -0.18991 \\
\hline & 0.020 & 0.08331 & 0.14881 & 3.39691 & 1.08721 & 0.53891 & -0.00011 & -0.15801 \\
\hline & 0.100 & 0.08331 & 0.14991 & 2.70091 & 1.08901 & 0.56861 & -0.00021 & -0.20951 \\
\hline
\end{tabular}

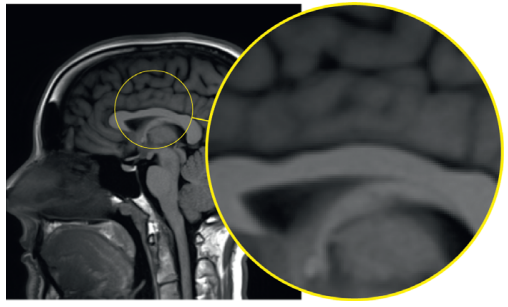

(a)

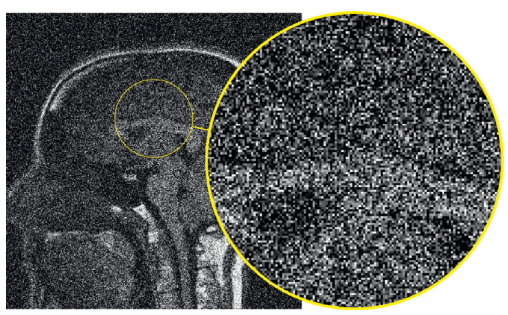

(d)

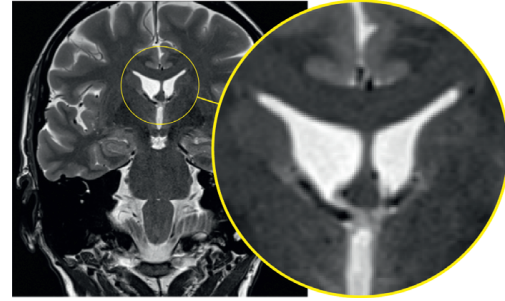

(b)

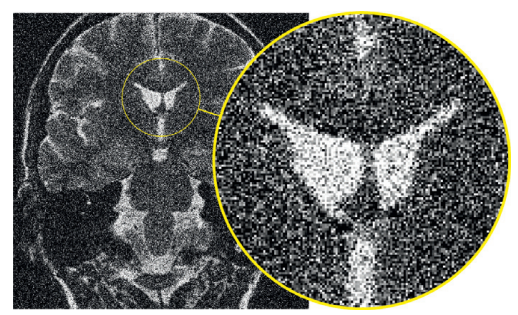

(e)

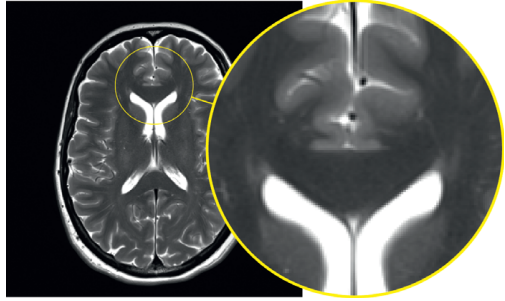

(c)

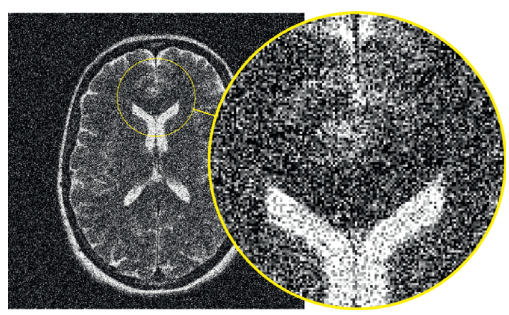

(f)

Figure 2: Continued. 


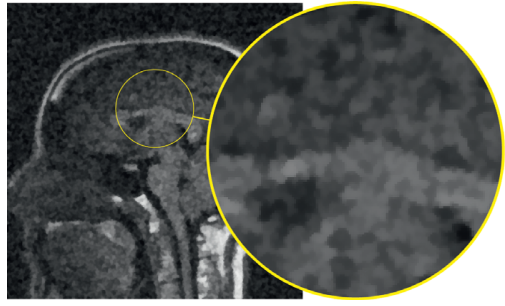

(g)

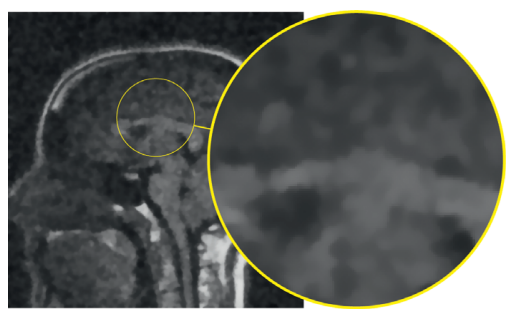

(j)

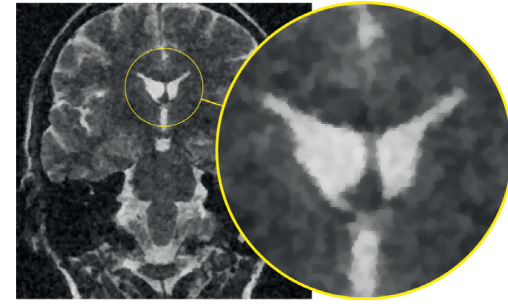

(h)

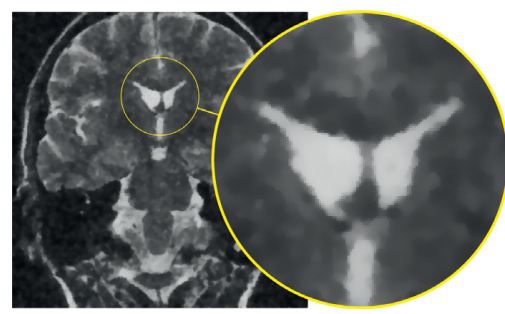

(k)

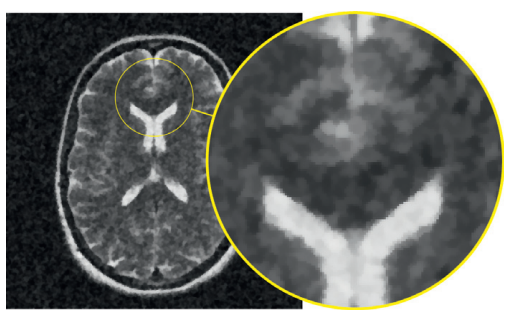

(i)

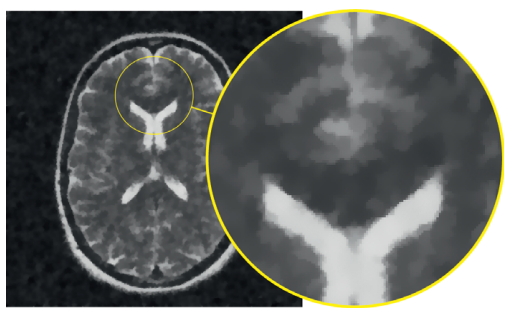

(l)

Figure 2: Visual comparison on different real images corrupted by Gaussian noise with $\sigma^{2}=0.1$ and restored images using both functions. (a) Original-Patient30. (b) Original-Patient50. (c) Original-Patient55. (d) Noisy-Patient30. (e) Noisy-Patient50. (f) Noisy-Patient50. (g) WZ-Patient30. (h) WZ-Patient50. (i) WZ-Patient55. (j) Proposed-Patient30. (k) Proposed-Patient50. (l) Proposed-Patient55.

the results from Table 1 prove that the suggested approach has higher values in SSIM, whereas the WZ model shows significant results in PSNR while $\sigma^{2}$-value increases.

\section{Conclusion}

This paper principally investigates the class of anisotropic diffusion partial differential equations related to image processing and analysis. The existence and uniqueness of weak solutions for this problem have been proven under sufficient conditions satisfied by $\phi$. A consistent and stable numerical approximation has been applied, and a discrete nonlinear filter has been tested and revealed its efficiency in the image restoration field.

\section{Data Availability}

No data were used to support this study.

\section{Conflicts of Interest}

The authors declare that there are no conflicts of interest regarding the publication of this paper.

\section{References}

[1] P. Perona and J. Malik, "Scale-space and edge detection using anisotropic diffusion," IEEE Transactions on Pattern Analysis and Machine Intelligence, vol. 12, no. 7, pp. 629-639, 1990.

[2] J. Weickert, Anisotropic Diffusion in Image Processing, Springer Vieweg Verlag, Wiesbaden, Germany, 1998.

[3] G. Aubert and P. Kornprobst, Mathematical Problems in Image Processing: Partial Differential Equations and the Calculus of Variations, Springer, New York, NY, USA, 2006.

[4] L. Wang and S. Zhou, "Existence and uniqueness of weak solutions for a nonlinear parabolic equation related to image analysis," Journal of Partial Differential Equations, vol. 19, no. 2, pp. 97-112, 2006.

[5] L. C. Evans, Partial Differential Equations, American Mathematical Society, Providence, RI, USA, 2010.

[6] N. Biranvand and A. Salari, "Energy estimate for impulsive fractional advection dispersion equations in anomalous diffusions," Journal of Nonlinear Functional Analysis, vol. 2018, pp. 1-17, 2018.

[7] K. P. P. Candito and U. Guarnotta, "Two solutions for a parametric singular p-laplacian problem," Journal of Nonlinear and Variational Analysis, vol. 4, pp. 455-468, 2020.

[8] Z. Feng and Z. Yin, "On weak solutions for a class of nonlinear parabolic equations related to image analysis," Nonlinear Analysis: Theory, Methods \& Applications, vol. 71, no. 7-8, pp. 2506-2517, 2009.

[9] P. Chen, "Existence and uniqueness of weak solutions for a class of nonlinear parabolic equations," Electronic Research Announcements in Mathematical Sciences, vol. 24, pp. 38-52, 2017.

[10] S. Li and P. Li, "Weak solutions for a class of generalised image restoration models," International Journal of Dynamical Systems and Differential Equations, vol. 8, no. 3, p. 190, 2018.

[11] T. Humphries, M. Loreto, B. Halter, W. O'Keeffe, and L. Ramírez, "Comparison of regularized and superiorized methods for tomographic image reconstruction," Journal of Applied and Numerical Optimization, vol. 2, pp. 77-99, 2020.

[12] M. Jourhmane, Méthodes numériques de résolution d'un problème d'électro-encéphalographie, Ph.D. thesis, University of Rennes 1, Rennes, France, 1993.

[13] G. Aubert and L. Vese, "A variational method in image recovery," SIAM Journal on Numerical Analysis, vol. 34, no. 5, pp. 1948-1979, 1997.

[14] J. Stoer and R. Bulirsch, Interpolation, pp. 37-144, Springer, New York, NY, USA, 2002.

[15] L. C. Evans and R. F. Gariepy, Measure Theory and Fine Properties of Functions, Taylor \& Francis Group, New York, NY, USA, 2015. 
[16] A. Tiarimti Alaoui and M. Jourhmane, "Existence and uniqueness of weak solutions for a new class of convex optimization problems related to image analysis," 2020.

[17] L. C. Evans, Weak Convergence Methods for Nonlinear Partial Differential Equations, American Mathematical Society, Providence, RI, USA, 1990.

[18] R. C. Gonzalez and R. E. Woods, Digital Image Processing, Prentice-Hall, Upper Saddle River, NJ, USA, 3rd edition, 2006.

[19] Z. Wang, A. C. Bovik, H. R. Sheikh, and E. P. Simoncelli, "Image quality assessment: from error visibility to structural similarity," IEEE Transactions on Image Processing, vol. 13, no. 4, pp. 600-612, 2004.

[20] F. Gaillard, "Normal brain mri," 2016.

[21] F. Gaillard, "Normal brain mri (tle protocol)," 2015.

[22] F. Gaillard, "Normal Mri Brain including MR venogram," 2017. 